\title{
Prospections archéologiques à grande échelle et géophysique pour les grands projets
}

\author{
Geophysics for large development projects and large scale archaeological \\ surveys
}

\author{
Dr. Michel DABAS ${ }^{1}$ \\ ${ }^{1}$ Chargé de Recherches au CNRS, ENS-AOROC UMR8546 ; Directeur Scientifique de Géocarta, PARIS \\ michel.dabas@geocarta.net
}

\begin{abstract}
RÉSUMÉ. La notion d'échelle est au cœur de la question de la prospection et particulièrement pour celui de la détection géophysique sur les grandes surfaces. Dans un premier temps, nous développons la place de la géophysique dans le diagnostic archéologique. Après avoir souligné l'utilisation maintenant reconnue de la méthode géophysique en archéologie programmée, nous analysons les arguments avancés pour justifier de son faible développement en archéologie préventive en France et en particulier sur les grands projets d'aménagement du territoire. Dans un second temps, une mise en perspective au niveau de l'Europe est faite. Enfin un historique de plusieurs expériences en France depuis 25 ans est dressé.

ABSTRACT. Scale is the key concept when dealing about survey and particularly for the geophysical survey over large areas. At first, the role of geophysics in rescue archaeology is described. After noticing the known role of geophysics in academic archaeology, we analyze the arguments given for its scarce use in preventive archaeology and in particular for large development projects in France. Secondly, a European perspective is made. Finally, a history of several experiences undertaken in France since 1995 is raised.

MOTS-CLÉS. Maillage, Géophysique, Echelle, Diagnostic archéologique.

KEYWORDS. Mesh, Geophysics, Scale, Archaeological evaluation.
\end{abstract}

\section{Introduction}

La notion d'échelle est au cœur du sujet de cette table ronde. En effet, elle définit de manière concomitante la taille des artefacts que l'on va chercher à détecter et les outils qu'il faudra déployer pour les détecter sur le terrain. L'archéologie a été depuis de nombreuses années un champ de recherche pour les notions de prospection. Nous ne parlerons ici que de la prospection géophysique appliquée spécifiquement à l'archéologie, mais il est clair que les concepts d'échelle s'appliquent aussi à d'autres types de prospection comme la prospection aérienne, pédestre et même le LiDAR pour citer une technique récente de cartographie du relief.

\section{Géophysique et diagnostic archéologique}

On peut commencer cet exposé en disant que tout problème de détection et cartographie archéologique relève a priori de la prospection géophysique. Certains pourraient objecter qu'il existe des cas où les méthodes géophysiques sont inopérantes, en citant par exemple les artefacts paléolithiques. Il est vrai qu'au stade technologique actuel, pour cette échelle, ce type d'objet a toutes les chances de passer inaperçu. Mais selon notre expérience, la géophysique permet dans ce cas d'apporter des informations cruciales sur le paleo-environnement et donc le raisonnement archéologique permettra alors d'inférer la probabilité d'existence de ces structures. Vouloir rejeter une technologie sous prétexte qu'elle n'est pas capable de "tout voir" renvoie forcément à la notion d'exhaustivité. Quel procédé, quelle technique permettrait de tout voir de la réalité archéologique ? Aucune assurément, sinon nous ne serions pas là pour en débattre dans cette table-ronde ! 
Il est plus prudent de dire qu'un ensemble de techniques doivent être employées conjointement dans le diagnostic archéologique afin de maximiser les capacités de détections et donc diminuer l'aléa de leur découverte pendant la phase travaux. Rappelons que le but du diagnostic est de donner le maximum d'informations aux services archéologiques de l'Etat pour étayer la demande de prescription. Rappelons aussi comme l'a fait Alain Tabbagh que les méthodes non destructives sont à privilégier puisque l'objet même de l'archéologie est d'étudier et de préserver notre patrimoine pour les générations futures.

La géophysique a été employée depuis les années 70 en France dans le cadre archéologique. Son développement a été lent et s'est cantonné tout d'abord à l'archéologie programmée, ce qui lui a permis de développer ses propres outils et expériences sur des surfaces généralement petites et où les questions archéologiques sont précisément définies. Son application à l'archéologie préventive est beaucoup plus récente et soulève un débat qui s'est cristallisé récemment autour de la mesure "14", du rapport rédigé par François Fichet de Clairfontaine et des avis divergents du CNRA (cf. article de L. Orengo dans ce recueil). Sans rentrer dans cette polémique nous souhaitons éclairer le débat en fonction de notre expérience.

La différence entre ces deux archéologies tient en effet à trois points au minimum: la notion d'échelle, la notion de temps et l'objet d'étude. Dire que la prospection géophysique n'est pas applicable à l'archéologie préventive alors qu'elle est devenue nécessaire et reconnue indispensable en archéologie programmée implique qu'au moins un de ces trois points - échelle spatiale, échelle temporelle, objetpose problème.

Tout d'abord la notion d'échelle spatiale: les surfaces qui ont été et sont encore étudiées par l'archéologie préventive (milliers d'hectares par an) sont significativement plus étendues que les surfaces étudiées en archéologie programmée. Or les mesures manuelles utilisées par les géophysiciens en archéologie jusque dans les années 2000 ne permettaient pas l'étude de telles surfaces. Depuis lors, l'apparition de méthodes trainées par des véhicules tout terrain a permis d'appréhender les grandes surfaces: toutes les méthodes manuelles, de l'électrique au radar en passant par les prospections magnétique et électromagnétique ont pu être automatisées et l'utilisation des systèmes de positionnement par satellite a permis un gain considérable de temps sur le terrain. Un seul opérateur peut maintenant prospecter de l'ordre de 3 à 10 ha par jour avec une résolution spatiale suffisante pour l'archéologie $(0,25 \mathrm{~m}$ à $0,50 \mathrm{~m})$. Bien évidemment le coût des prospections a diminué en proportion et l'argument financier ne peut plus être invoqué pour écarter les méthodes géophysiques.

La notion de temps a aussi été avancée un moment pour écarter les méthodes géophysiques de la boite à outil de l'archéologue « urgentiste ». Pour les mêmes raisons que précédemment, les avancées technologiques récentes permettent de couvrir de grandes surfaces rapidement. Pour des chantiers particulièrement importants auxquels nous avons été confrontés, comme une autoroute ou le tracé d'un pipeline par exemple et où le temps imparti est très limité, la mise en œuvre de plusieurs équipes en parallèle permet de réduire d'autant le temps terrain et de respecter les contraintes imposées par le Maitre d'ouvrage. Rappelons aussi que l'aspect non destructif des méthodes géophysiques permet de les utiliser en amont des projets, même avant l'acquisition foncière, ce qui reste un atout considérable : cette connaissance permet éventuellement de modifier le projet et donc de protéger les structures détectées.

L'objet reste maintenant probablement le dernier point qui semble poser encore problème. L'outil géophysique n'est pas capable de détecter tout ce que peut détecter l'archéologue de visu dans un sondage, mais l'inverse est aussi vrai. Nous avions évoqué au départ de cet exposé le cas des structures paléolithiques, nous aurions tout aussi bien pu évoquer celui de trous de poteaux de faible dimension. Mais les choses ne sont pas si facilement comparables: l'archéologue fouilleur est habitué à appréhender la réalité archéologique au travers de ses propres sens (un physicien parlerait de capteurs): visuellement, par une différence de coloration, ou par exemple tactilement, par une (c) 2017 ISTE OpenScience - Published by ISTE Ltd. London, UK - openscience.fr 
différence de dureté d'un niveau de sol sous la truelle. Le prospecteur géophysicien appréhende la même réalité archéologique par une série de mesures (on parle de quantification). Il sera capable de traduire en chiffres non seulement les paramètres connus de l'archéologue comme les différences de couleur ou de dureté, mais aussi d'autres paramètres peu familiers à nos sens comme une grandeur électrique ou magnétique par exemple. Et ces paramètres peuvent être tout aussi importants pour l'archéologue pour délimiter par exemple une aire d'activité d'un secteur artisanal où à l'œil rien n'est visible. De la même façon que l'archéologue de terrain connait ses propres limites de détection (par exemple est-ce que les fossés seront visibles dans tel type de terrain lorsque le terrain est humide), le prospecteur connait les limites des techniques qu'il utilise puisqu'il dispose de programmes de simulation qui lui permettent de prédire la réponse d'une structure enfouie en fonction de ses caractéristiques, de son environnement et de la technique de prospection utilisée.

Rappelons aussi comme l'a fait Alain Tabbagh dans son exposé que les méthodes géophysiques sont non destructives, respectant par là-même le traité de la Valette. Ce traité, qui suit pour partie des règles édictées déjà en Angleterre (PPG15 et PPG16), reconnait l'archéologie comme une ressource non renouvelable et stipule que les méthodes non-destructives doivent être utilisées autant que possible (Convention européenne pour la protection du patrimoine archéologique, Traité $\mathrm{N}^{\circ} 143$ de La Valette du 16 Janvier 1992, ratifié par la France en Juillet 1995, Article 3: "En vue de préserver le patrimoine archéologique et afin de garantir la signification scientifique des opérations de recherche archéologique, chaque Partie s'engage: à mettre en œuvre des procédures d'autorisation et de contrôle des fouilles, et autres activités archéologiques, afin: de prévenir toute fouille ou déplacement illicites d'éléments du patrimoine archéologique; d'assurer que les fouilles et prospections archéologiques sont entreprises de manière scientifique et sous réserve que: des méthodes d'investigation non destructrices soient employées aussi souvent que possible).

\section{Situation actuelle de la prospection géophysique dans les grands projets}

On peut considérer que les techniques géophysiques sont maintenant opérationnelles pour les grandes surfaces et dans le cadre de l'archéologie préventive : des pays comme l'Angleterre, l'Allemagne, la Suède, le Danemark, la Hongrie, la Slovénie l'utilisent à grande échelle. Cette reconnaissance a même été formalisée au travers de manuels dont le plus ancien est celui d'English Heritage publié en 1995 " Geophysical Survey in Archaeological Field Evaluation" et réédité en 2008. Cette publication a défini les règles pour le déroulement d'une prospection géophysique et le rapport correspondant, et fournit un guide pour le choix de la méthode géophysique et son utilisation sur le terrain. Il y est indiqué en particulier que « la prospection géophysique doit faire partie des principales techniques utilisées lors de l'évaluation d'un site, et sa contribution doit toujours être envisagée à chaque instant, lorsqu'un projet d'aménagement est lancé ». Nous renvoyons aussi au livre de Armin Schmidt " Geophysical Data in Archaeology: A Guide to Good Practice (2nd edition. Oxford and Oakville: Oxbow Books). Plus récemment, l'EAC (Europae Archaelogiae Consilium) a émis le guide probablement le plus complet à la date d'aujourd'hui : "EAC Guidelines for the Use of Geophysics in Archaeology" (135 pages).

\section{Historique de l'introduction des méthodes géophysiques pour les grands projets}

Au niveau français, nous souhaitons témoigner de l'évolution des méthodes géophysiques depuis nos premières expériences en 1995 sur des autoroutes (Dabas, 2016).

Les premières tentatives de prospection que nous avons effectuées en France sur de grandes surfaces ont été conduites sur l'autoroute A77 en 1995 pour le compte de la Société des Autoroutes Paris-RhinRhône à la demande de Mme Frère-Sautot (SAPRR) et sous le contrôle de la DRAC du Centre et de Bourgogne. Le but était la détection d'un ensemble de ferriers probablement d'époque romaine sur le futur tracé de l'autoroute entre Dordives et Cosne-sur-Loire. La particularité de ce chantier outre sa 
surface (bande de $300 \mathrm{~m}$ de large sur $1050 \mathrm{~m}$ de longueur) était qu'il était entièrement en sous-bois. Nous avons cartographié manuellement cette surface en utilisant un appareil très simple (appareil EM15, Geonics) capable de mesurer la susceptibilité magnétique, paramètre très discriminant pour la détection de scories métalliques. Les mesures ont été faites selon des profils parallèles espacés de 10 mètres et dans deux directions orthogonales. Malgré cet échantillonnage que l'on qualifie de large (maille large), il a été possible de cartographier les limites de quatre sites en utilisant des méthodes d'interpolations spatiale ad hoc (Dabas, 1999a) : figure 1. Les simulations sur ordinateur ont montré qu'on aurait pu porter l'espacement entre les profils à $20 \mathrm{~m}$ voire à $50 \mathrm{~m}$ le long des profils et limiter les profils à une seule direction. Cette première expérience positive a eu trois conséquences: i) avant même que les fouilles ne commencent, le trajet de l'autoroute a pu être modifié afin de préserver une partie des sites; ii) SAPRR a demandé de poursuivre cette étude à une portion plus importante d'une dizaine de kilomètres (A77 Sud, portion Briare Cosne-sur-Loire), iii) la méthode de cartographie par la susceptibilité magnétique a été utilisée pour d'autres autoroutes (A89, A20 et A66) et a été l'objet d'un sujet de thèse piloté par l'AFAN et l'Université Paris VI (Marmet, 2000).

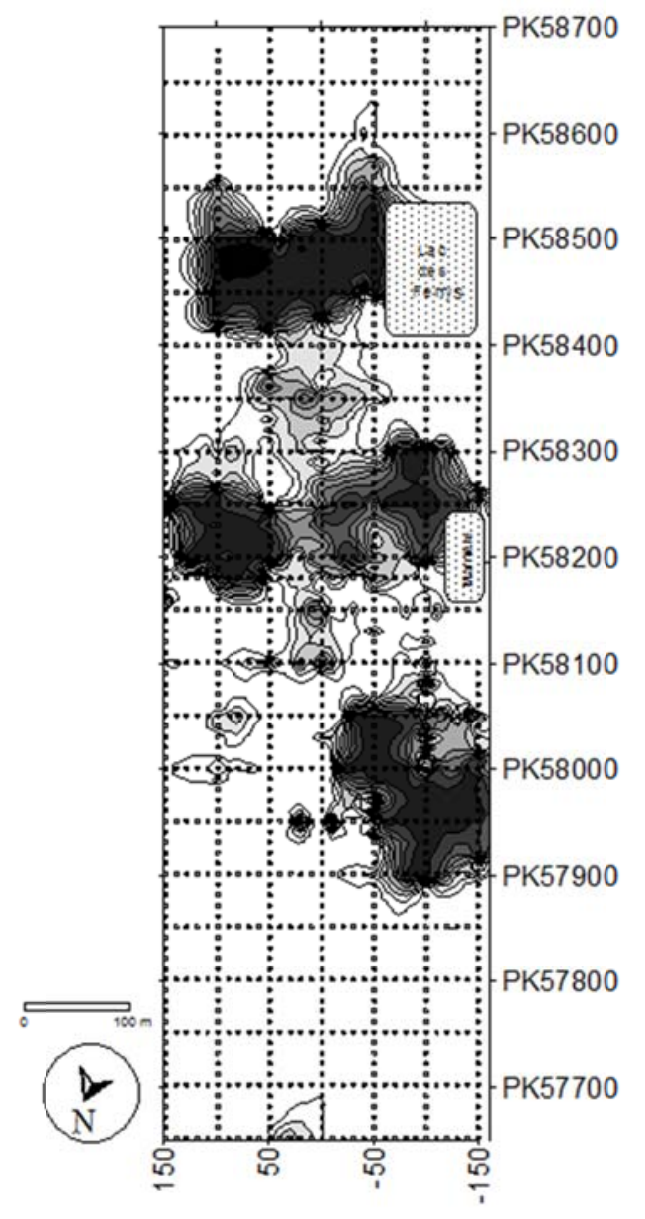

Figure 1. Carte de susceptibilité magnétique apparente, Autoroute A77

En 1995, nous avons été en charge du diagnostic non invasif de l'A77 Sud sur 15km de longueur et $100 \mathrm{~m}$ de largeur. Une des originalités de ce projet a été d'utiliser à l'époque des Systèmes d'Information Géographiques (GRASS et Idrisi) pour le pilotage global du projet (Chazaly et Dabas, 1997; Dabas 1999b). Comme pour le projet précédent des ferriers, la cartographie de la susceptibilité magnétique a été effectuée sur tout le tracé (appareil MS2b Bartington, maille de $10 \times 25 \mathrm{~m}$ ). Les moyens manuels de l'époque ne nous permettaient pas d'effectuer des prospections électriques et magnétiques sur tout le tracé. L'idée était de ne les déclencher que sur les surfaces où une augmentation anormale de la susceptibilité était observée. Plusieurs autres informations avaient été utilisées : photographies aériennes, cadastre napoléonien, missions photogrammétriques, extraits de la base de donnée DRACAR, résultats des prospections pédestres que nous avions faites sur toutes les surfaces labourées, 
carte pédologique faites par nos soins. Il a été possible en croisant ces informations de créer une carte du risque d'érosion/comblement des sites potentiels. Le résultat a été la découverte de 21 indices de site (seuls deux étaient connus avant nos travaux). La prospection mécanique faite après notre travail a confirmé l'existence des sites. Les travaux ultérieurs ont porté sur l'utilisation de la susceptibilité magnétique comme marqueur potentiel de la présence de sites sur plusieurs projets d'autoroute (A89, A20 et A86). Rappelons qu'à l'époque c'était la seule méthode qui pouvait être appliquée à l'intégralité de ces grandes surfaces dans un temps raisonnable. Les travaux de thèse d'Eric Marmet ont prouvé que cette hypothèse était trop simpliste: si des sites, même de taille modeste, peuvent être détectés par cette augmentation, il existe aussi des causes naturelles d'augmentation de la susceptibilité magnétique. Par exemple sur 9 sites détectés par les tranchées mécaniques sur une autoroute, 7 ont été détectés par la susceptibilité. Les autres augmentations de susceptibilité n'ont pas été interprétées comme dues à l'existence de site ('faux positif'). Parmi les 2 sites non détectés par la susceptibilité, E. Marmet note " ces deux sites ont été d'abord détectés par prospection pédestre et la probabilité de les détecter par les tranchées mécaniques était très faible".

Il est clair que la mesure de la susceptibilité magnétique a fait ses preuves en archéologie mais que son utilisation seule n'est pas suffisante pour la détection de tous les indices de site et ce pour trois raisons: les appareils des années 90 étaient trop lents pour qu'on puisse envisager une utilisation en continu et à large échelle, il faut disposer d'une connaissance pédologique importante afin d'éliminer les causes naturelles d'augmentation de la susceptibilité magnétique et éliminer donc les faux-positifs; enfin la profondeur d'investigation des appareils était très faible (quelques dizaines de centimètres).

Pour toutes ces raisons, nous avons voulu développer à Géocarta depuis 2001 les autres méthodes qui avaient fait leur preuve en archéologie, à savoir dans un premier temps les méthodes électriques et magnétiques et dans un second temps les méthodes électromagnétiques à la fois basse fréquence et haute-fréquence (RADAR). Le but était de pouvoir disposer de systèmes automatisés capables de couvrir des surfaces de plusieurs hectares par jour et ce avec une résolution spatiale correcte pour l'archéologie ( 0,50 à $0,25 \mathrm{~m}$, soit 40000 à 160000 mesures/ha). Pour ce faire, deux obstacles devaient être surmontés : le passage d'une mesure statique - comme dans les procédés manuels- à une mesure en dynamique et un positionnement dynamique (chaque mesure en continu doit être associée à un positionnement planimétrique au mieux décimétrique). Les progrès sur les capteurs, leur électronique, l'acquisition et le traitement des données a permis en l'espace de quelques années de développer avec succès ces technologies : ARP $\odot$ pour la mesure de la résistivité, AMP pour la mesure magnétique et EMP pour la mesure électromagnétique (figure 2). Nous avons commencé à les appliquer à des grandes surfaces pour l'archéologie à partir de 2006.
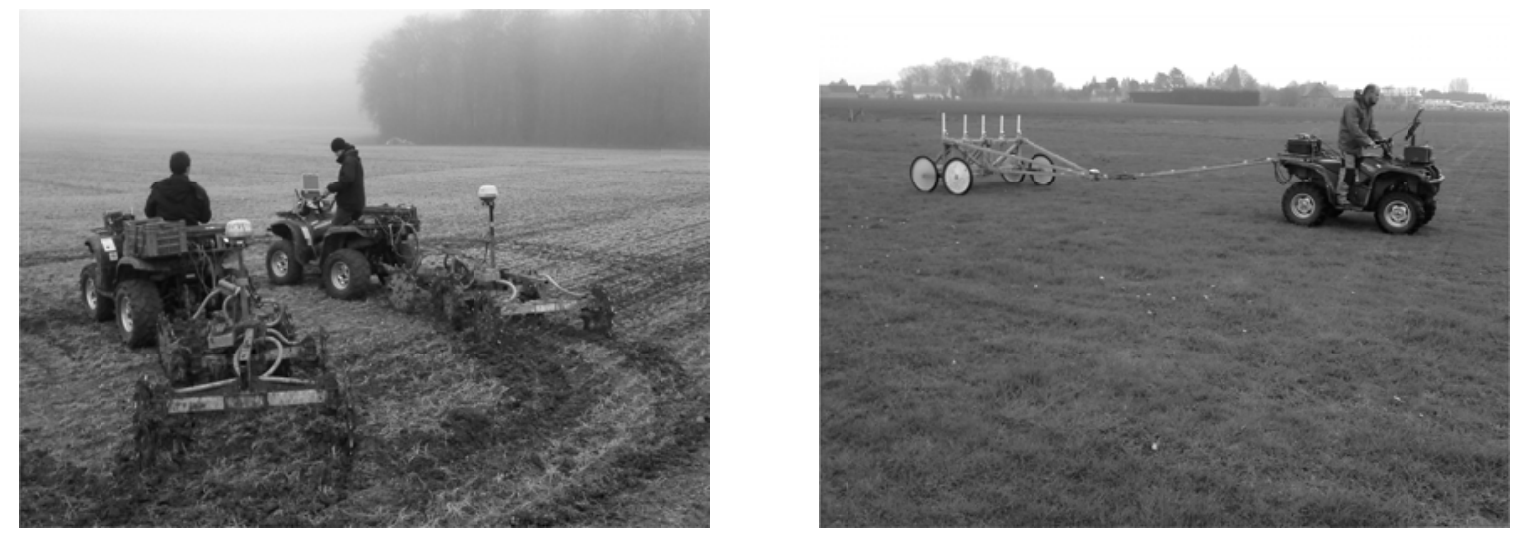

Figure 2. Méthode électrique automatisée $A R P \otimes$ (gauche) et magnétique $A M P($ droite)

La première étude a porté sur le projet d'aménagement de la ZAC de Veimars à côté de l'aéroport Charles-de-Gaulle sur 50ha (client: GSE Prologis, sous la supervision de la DRAC ÎdF M. Gouedo). La prospection a été faite en seulement 2 semaines en utilisant les trois techniques. La carte, figure 3, 
montre les résultats avec une résolution spatiale de $0,5 \times 0,5 \mathrm{~m}$. Un seul site a été détecté, ce qui est une situation anormale pour cet endroit et sur une telle surface. Le diagnostic en campagne de tranchées mécaniques (opérateur: SDAVO) n'a pas trouvé d'autres sites. Par contre lors de la phase des travaux, un second indice de site a été détecté (signalé comme "un vide protégé par une arche") et détruit immédiatement car non signalé lors de la phase diagnostic. Une analyse a posteriori des cartes géophysiques a pu montrer que l'anomalie avait été détectée mais non interprétée.
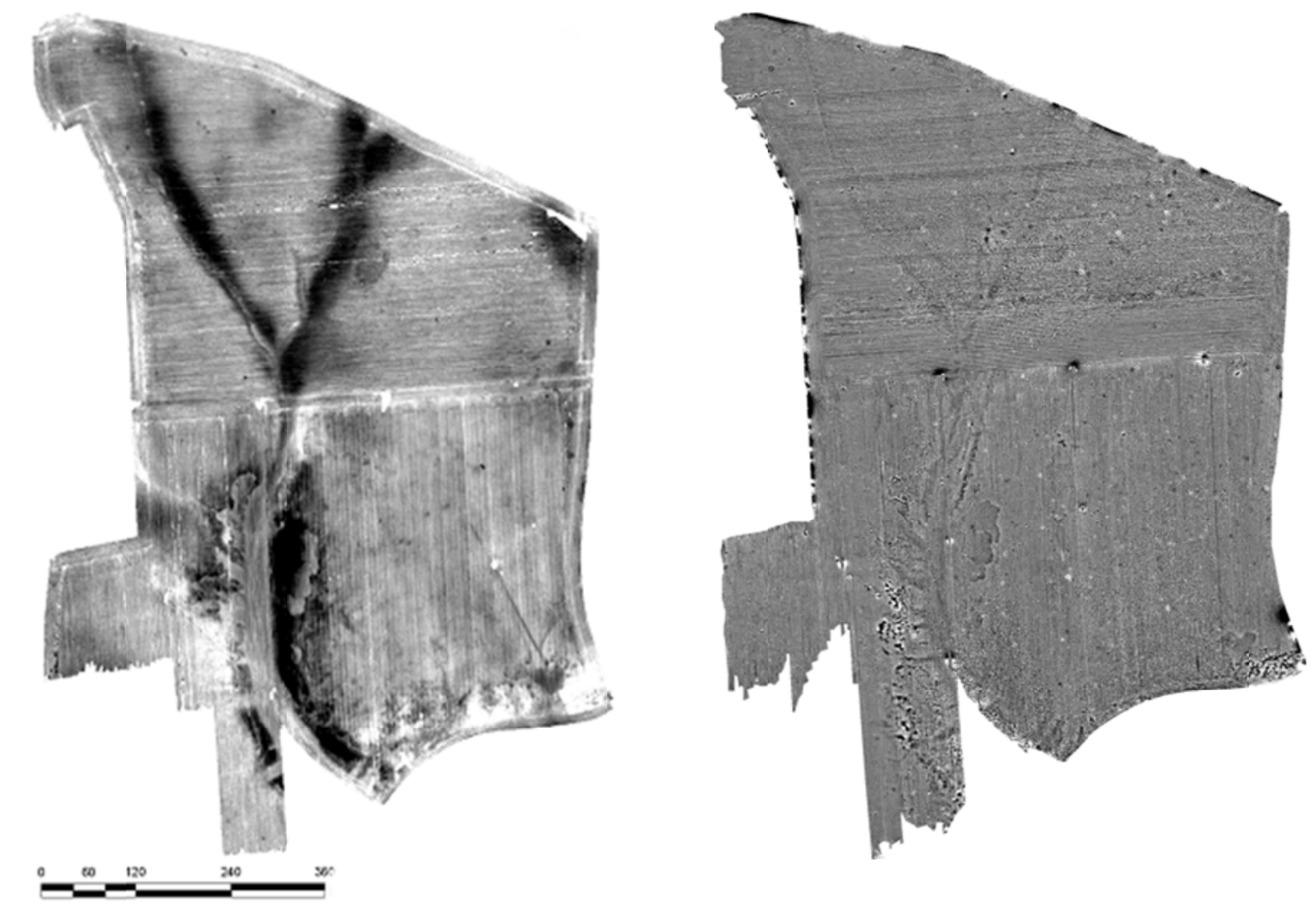

Figure 3. Carte de résistivité (gauche) et magnétique (droite) ZAC de Veimars

Pendant les dix dernières années, nous avons été amenés à travailler sur dix projets de taille importante ( $>20 \mathrm{ha}$ ), la plupart correspondant à des projets de ZAC ou des infrastructures linéaires (autoroutes, déviations routières ou servitudes enterrées). Nous avons toujours préconisé au minimum l'emploi de deux méthodes géophysiques à savoir l'électrique et le magnétique et, pour les sites plus profonds $(>2 \mathrm{~m})$, nous proposons les méthodes électromagnétiques. Nous ne développerons pas ici le projet de l'autoroute Milan-Brescia en Italie (Consortium BREBEMI) qui est développé par S. Campana dans ce recueil et qui correspond au projet le plus abouti d'une démarche visant à l'étude non destructive non d'un site ou d'un ensemble de sites, mais de tout l'environnement sur plus de 430ha. Ce projet s'inscrit dans la ligne du projet européen 'Emptyscapes' qui vise à étudier tout l'environnement des sites potentiels comme un continuum (Bintliff, 2000) (Campana S., 2016). Une seule carte est montrée à titre d'exemple (figure 4). Cette carte magnétique montre de nombreuses structures liées à l'environnement (système de paléocheneaux en tresse) mais aussi archéologiques dans le coin sud ouest (nécropole). 


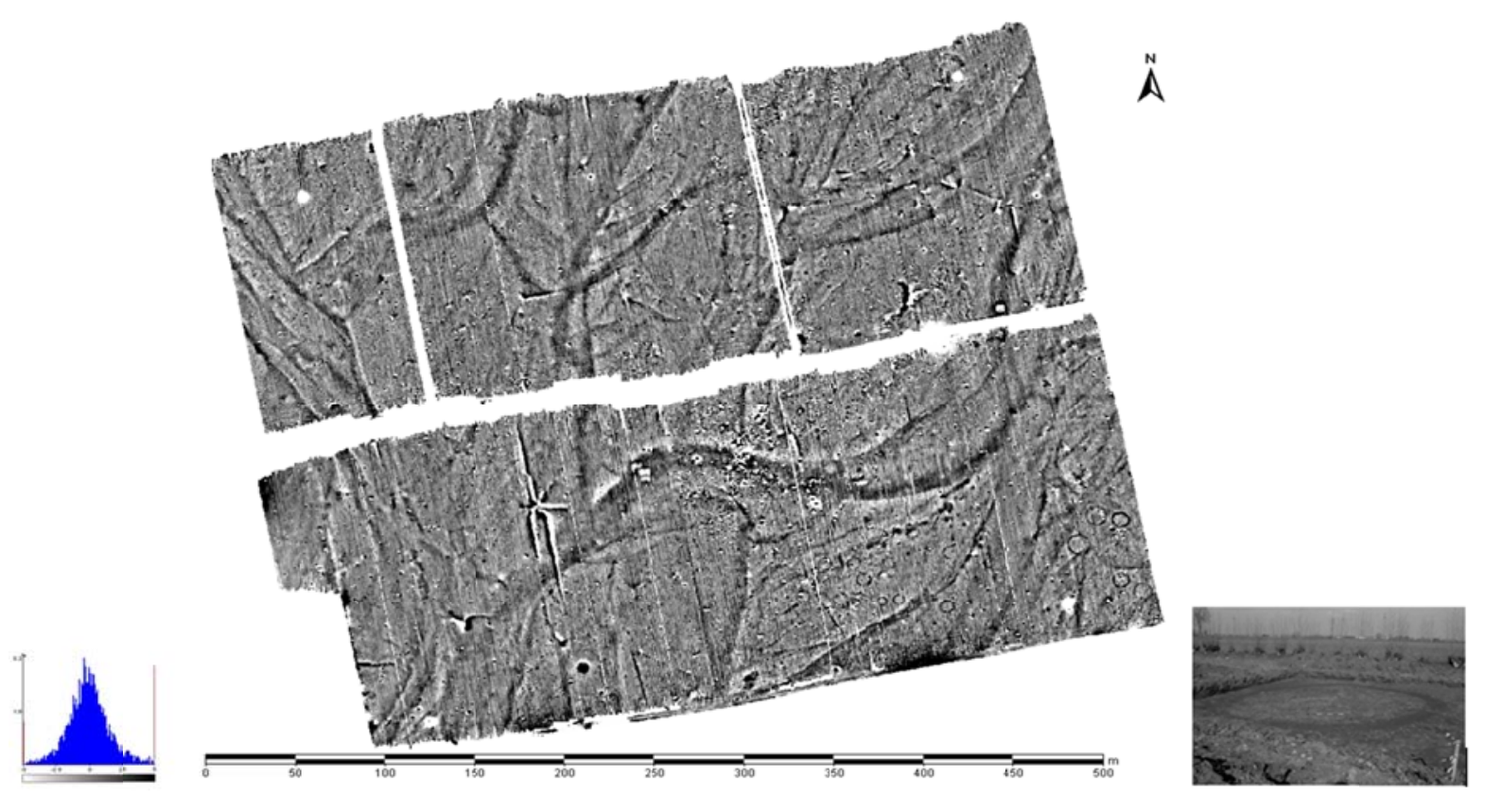

Figure 4. Carte de magnétisme, autoroute Milan-Brescia (paleo-cheneaux et nécropole)

Nous finirons par le diagnostic le long d'un fuseau de $22 \mathrm{~km}$ de long pour la construction d'une tranchée pour l'enfouissement d'une ligne électrique haute tension en 2011 (client : RTE, suivi SRA Picardie). Le problème sur ce dossier était plus d'ordre géotechnique qu'archéologique. Il s'agissait de rechercher l'occurrence de vides liés pour la plupart à la présence de tranchées de la première guerre mondiale sur cette zone qui a correspondu à la ligne de front lorsqu'elle s'est stabilisée pendant l'hiver 1914-1915. Comme le projet d'enfouissement ne concernait qu'une largeur de 1mètre, les services de l'Etat n'ont pas demandé de diagnostic archéologique par tranchées. Néanmoins, et en accord avec RTE, il a été décidé de prospecter un fuseau beaucoup plus large de $100 \mathrm{~m}$ et en utilisant une maille fine $(0,5 \mathrm{mx} 0,5 \mathrm{~m})$. Les méthodes électrique et magnétique ont été employées ainsi que la méthode électromagnétique pour atteindre une profondeur d'investigation de 6 mètres (pratiquement on dispose de 6 profondeurs d'investigation entre $0,5 \mathrm{~m}$ et $6 \mathrm{~m}$ de profondeur). Comme pour les projets cités précédemment, un SIG spécifique est utilisé comme outil à la fois pour l'acquisition sur le terrain et pour le traitement de l'information. La méthode de résistivité a permis de détecter tous les artefacts liés à cette zone de bataille (tranchées, impacts de bombes, chemins d'accès). De nombreuses éléments pyrotechniques (bombes, restes de bombes, etc.) ont été retrouvés. Ces éléments ont été recoupés avec des cartes des Forces Alliées. La résolution des cartes a permis aussi de détecter des sites archéologiques plus anciens. Aucun de ces sites n'a donné lieu à un diagnostic ou à une fouille eu égard à l'impact très faible de la tranchée d'enfouissement sur ces structures. Le tracé définitif a pu finalement être défini à l'intérieur du fuseau des 100 mètres en prenant en compte toutes les informations du diagnostic non-destructif. De plus, RTE a pu minimiser les effets indésirables de l'enfouissement mécanique dans les champs (pose en une seule passe, trancheuse sur chenille, pose de palplanches, séparation des terres, etc...).

\section{Conclusion}

Nous avons vu à travers ces différents exemples une utilisation des moyens géophysiques en archéologie préventive. La question n'est plus de savoir si leur coût est prohibitif ou s'il faut ou non utiliser ces outils. La question est de savoir quand, où et avec quelles autres méthodes. Nous pensons que l'outil géophysique peut apporter une couche d'information à la fois utile pour l'aménageur aspects pédologiques, géotechniques, hydrologéogiques - et pour l'archéologue -détection des indices de site mais aussi et surtout connaissance de l'environnement, vu non pas comme une entité séparée mais comme un tout. Notre expérience en aménagement du territoire depuis 25 ans nous amène à 
penser que le diagnostic archéologique, et de manière plus générale la phase archéologique, n'est qu'une petite partie de l'évaluation du risque de tout projet industriel, qu'il s'agisse d'un petit projet de lotissement ou de la construction d'un canal. Nous avons développé une approche heuristique où la connaissance des risques (donc leur coût) est mutualisée entre les différents acteurs d'un projet. Cette approche n'a été possible que grâce à la connaissance des différents acteurs du développement du territoire, au développement des techniques rapides de prise d'information sur le terrain et à l'utilisation d'outils informatiques (web-SIG) permettant d'échanger et partager cette information. Le dernier exemple (RTE) a permis de montrer l'importance de ces informations partagées dans ce cas entre les géotechniciens et les personnes en charge du risque pyrotechnique. Nous finirons par dire que l'archéologue dont le travail in fine est payé par l'aménageur n'est pas seul dans la boucle.

\section{Bibliographie}

BINTLIFF, J. 2000. "Beyond dots on the map: future directions for surface artefact survey in Greece". In: Bintliff J., Kuna M., Venclova N., The future of surface artefact survey in Europe, Sheffield Academic Press, Sheffield.

BLOUET, Y. 1994. "Essais de comparaison de différentes méthodes d'étude archéologiques préalables". Les Nouvelles de l'Archéologie, 58:17-19.

CAMPANA, S. 2009. "Archaeological Site Detection and Mapping: some thoughts on differing scales of detail and archaeological 'non-visibility', in Campana S., S.Piro, Seeing the unseen, Taylor \& Francis, The Netherlands.

CAMPANA , S., DABAS, M. 2011. "Archaeological Impact Assessment: The Brebemi Project (Italy)", Archaeological Prospection, 18, 2:139-148.

CAMPANA, S. 2016. "Towards mapping the archaeological continuum. New perspectives and current limitations in Planning Led Archaeology in Italy", Looking for the future, Caring for the past, Preventive Archaeology in Theory and Practice, ed. F. Boschi, Bononia University Press 2006, in press.

CHAZALY, B., DABAS M. 1997. "SIG et Détection archéologique", Revue XYZ, 72: 47-51.

DABAS, M. 1999a. "Contribution de la prospection géophysique à large maille et de la géostatistique à l'étude des tracés autoroutiers. Application aux ferriers de la Bussière sur 1'A77". Revue d'Archéométrie, 5:7-32.

DABAS, M. 1999b." Diagnostic et évaluation du potentiel archéologique dans le cadre des tracés linéaires : apport des Systèmes d'Information Géographiques". Revue d'Archéométrie, 23:5-16.

DABAS, M., BLIN, O., BENARD, C. 2005. "Les nouvelles techniques de résistivité électrique employées dans la prospection de grandes surfaces en archéologie". Les Nouvelles de l'Archéologie, 101, 4:24-32.

DABAS, M. 2009. "Theory and practice of the new fast electrical imaging system ARP®". Seeing the Unseen, Geophysics and Landscape Archaeology, Campana and Piro eds., CRC Press, Taylor and Francis Group, 105-126.

DABAS, M. 2016. "Preventive Archaeology in France and the contribution of extensive geophysics: from ARP $\odot$ to webGIS",", Looking for the future, Caring for the past, Preventive Archaeology in Theory and Practice, ed. F. Boschi, Bononia University Press 2006, in press.

FERDIERE, A 2006. « La prospection », collection « archéologiques », éditions errance, Paris.

HULIN, G., PRILAUX, G., TALON, M. 2014. Intégration de la géophysique à un projet archéologique d'envergure. L'exemple du projet canal Seine-Nord-Europe., Revue Archéologique de Picardie, 1 : 245-260.

MARMET E., 2000. «Cartographie à large maille de la susceptibilité magnétique du sol, pour une évaluation archéologique sur les grands tracés ». Thèse Université Pierre et Marie Curie

MARMET E., BEST, C., TABBAGH, A., 2002. «Prospection systématique par sondages à la pelle mécanique: limites liées à la probabilité de découverte de sites archéologique ». Revue d'Archéométrie, 26 : 11-21.

SIMON, F.-X. 2012. «L'apport de l'outil géophysique pour la connaissance et la caractérisation des sites en archéologie préventive, méthodes et perspectives : exemple en Alsace ». Thèse Université Pierre et Marie Curie. 